\title{
PROTOCOLO DE AVALIAÇÃO DO FRÊNULO DA LÍNGUA EM BEBÊS
}

\section{Tongue frenulum evaluation protocol in babies}

\author{
Roberta Lopes de Castro Martinelli ${ }^{(1)}$, Irene Queiroz Marchesan (2), \\ Antonio de Castro Rodrigues ${ }^{(3)}$, Giédre Berretin-Felix ${ }^{(4)}$
}

\section{RESUMO}

Objetivo: apresentar uma proposta de protocolo de avaliação do frênulo da língua em bebês com escores. Método: inicialmente foi realizada uma revisão da literatura relacionada, consultando as bases de dados Web of Science, Pubmed, Embase, All Evidence-Based Medicine Reviews e Scielo, bem como livros textos das áreas de anatomia, odontopediatria e fonoaudiologia. Todos os aspectos julgados relevantes nos trabalhos encontrados foram incluídos na versão inicial, que foi submetida à avaliação de três fonoaudiólogas especialistas em motricidade orofacial, sendo obtida a versão consenso, que foi aplicada em 10 bebês nascidos a termo buscando verificar a exequibilidade do instrumento. Resultados: com base na literatura consultada e no estudo piloto, foi elaborado o protocolo de avaliação do frênulo da língua em bebês. A primeira parte é composta por história clínica contendo questões gerais de identificação e questões específicas sobre antecedentes familiais e amamentação. A segunda parte é composta pelo exame clínico, constituído de avaliação anatomofuncional e avaliação das funções orofaciais. Conclusões: o protocolo de avaliação do frênulo da língua em bebês foi desenvolvido a partir de uma proposta teórica e depende de sua aplicabilidade para se configurar como um teste validado. Espera-se que o mesmo possa auxiliar os profissionais da saúde a avaliar e diagnosticar as variações anatômicas do frênulo e sua possível interferência na amamentação, norteando condutas eficazes e promovendo uma prática baseada em evidências. A segunda fase deste trabalho deve compreender a pesquisa experimental e a análise estatística dos dados.

DESCRITORES: Avaliação; Protocolos; Freio Lingual; Amamentação; Sucção; Fonoaudiologia

\section{INTRODUÇÃO}

A língua é um órgão especializado localizado na cavidade oral, participando ativamente das funções de sucção, deglutição, mastigação e fala. Possui, em sua face inferior, uma pequena prega de membrana mucosa que a conecta ao assoalho da boca, sendo denominada frênulo da língua ${ }^{1,2}$.

(1) Fonoaudióloga, Mestranda em Ciências Odontológicas Aplicadas, Faculdade de Odontologia de Bauru, Universidade de São Paulo, Brasil.

(2) Fonoaudióloga, Diretora do CEFAC Pós-Graduação em Saúde e Educação, Doutora em Educação pela UNICAMP, Brasil.

(3) Professor Associado, Departamento de Ciências Biológicas, Faculdade de Odontologia de Bauru, Universidade de São Paulo, Brasil.

(4) Professora Associada, Departamento de Fonoaudiologia, Faculdade de Odontologia de Bauru, Universidade de São Paulo, Brasil.

Conflito de interesses: inexistente
O frênulo possibilita ou interfere na livre movimentação da língua. Quando não ocorre a apoptose completa do frênulo, durante o desenvolvimento embrionário, o tecido residual que permanece pode limitar os movimentos da língua, podendo levar à anquiloglossia.

Anquiloglossia, portanto, é uma anomalia oral congênita, que pode ocorrer de forma total ou parcial, limitando a mobilidade de língua em graus variados e podendo interferir nas funções orais ${ }^{3-24}$.

Diagnosticar a anquiloglossia total não é difícil, pois ela é muito visível; mas diferenciar as variações anatômicas do frênulo requer conhecimento bastante aprofundado da anatomia da língua e do assoalho da boca para identificar se os achados anatômicos podem comprometer a movimentação da língua e consequentemente, as funções orais.

Não há consenso nos critérios utilizados para a avaliação e classificação anatômica do frênulo da língua, o que pode justificar a grande variação, 
entre $0,88 \%$ e $12,8 \%$, nos índices de incidência de anquiloglossia ${ }^{3-12}$. Na literatura consultada, apenas um protocolo foi proposto para avaliação do frênulo em bebês até seis meses de idade ${ }^{13}$. Este protocolo apresenta vários itens referentes à aparência do frênulo e propõe a avaliação dos movimentos da língua via estimulação de reflexos, e da função de sucção não nutritiva. Entretanto, Ricke et al ${ }^{14}$ relatam limitações desse protocolo para identificar quais crianças com língua presa apresentam riscos para dificuldades na amamentação. Knox ${ }^{16}$ relatou, ainda, que esse instrumento de avaliação atualmente não é muito utilizado, possivelmente devido à sua complexidade.

Em bebês, a amamentação está diretamente relacionada com as funções de sucção e deglutição, coordenadas com a respiração. Em ambas, a participação dos movimentos da língua é fundamental. Sendo assim, qualquer restrição à livre movimentação da língua pode resultar no comprometimento das funções, dificultando a amamentação, porém, não foram encontrados na literatura estudos acerca dessa relação. Essa dificuldade para amamentar pode levar ao desmame precoce e/ou baixo ganho de peso, comprometendo o desenvolvimento dos bebês ${ }^{5,11,13-23}$.

O objetivo desse trabalho foi apresentar uma proposta de protocolo de avaliação do frênulo da língua em bebês com escores.

\section{MÉTODO}

Inicialmente foi realizada uma revisão da literatura relacionada, sendo consultadas as bases de dados Web of Science, Pubmed, Embase, All Evidence-Based Medicine Reviews e Scielo, usando os seguintes termos em português: freio lingual, anquiloglossia, frenectomia lingual, frenotomia lingual, sucção nutritiva; e dos seguintes termos em inglês: tongue-tie ankyloglossia, lingual frenulum, lingual frenum, lingual frenectomy, frenotomy $e$ sucking. Foi realizada também uma busca nas referências dos artigos encontrados e foram consultados livros textos das áreas de anatomia, odontopediatria e fonoaudiologia.

Para identificar os artigos de interesse, a pesquisa inicial foi realizada pelo título e resumo. Foram excluídos os artigos cujo tema não era pertinente à pesquisa, as cartas ao editor e os editoriais. Posteriormente, os artigos selecionados foram lidos na íntegra e analisados quanto aos seus objetivos, ao número e gênero dos participantes, ao método do estudo, e quanto aos resultados obtidos.

Todos os aspectos anatomofuncionais do frênulo e da língua e as propostas de avaliação da sucção nutritiva julgados relevantes nos trabalhos encontrados foram incluídos na versão inicial, que foi submetida à avaliação de três fonoaudiólogas especialistas em motricidade orofacial, sendo obtida a versão consenso, que foi aplicada em 10 bebês nascidos a termo buscando verificar a exequibilidade do instrumento.

No projeto piloto foram realizadas filmagens das avaliações anatomofuncional e das funções orofaciais de todos os bebês.

$\mathrm{Na}$ avaliação anatomofuncional foi observada a postura dos lábios em repouso e a tendência do posicionamento da língua durante o choro. Por meio da elevação das margens laterais da língua com os dedos indicadores direito e esquerdo enluvados da avaliadora, foi observado se era possível visualizar ou não o frênulo; se fosse possível visualizá-lo, era verificada sua espessura e sua fixação na língua e no assoalho da boca.

Para a avaliação das funções orofaciais foram filmadas a sucção não nutritiva e a sucção nutritiva.

A avaliação da sucção não nutritiva foi avaliada com a introdução do dedo mínimo enluvado na boca do bebê para sugar durante 2 minutos. Foi observada a movimentação e o canolamento da língua, bem como a força da sucção.

Para a avaliação da sucção nutritiva, foi utilizado um oxímetro de pulso para medir a frequência cardíaca e o nível de saturação de oxigênio no início e no final da avaliação.

As características do mamilo materno e a "pega" do mesmo pelos bebês foram observadas.

$\mathrm{Na}$ avaliação da sucção propriamente dita, foi observado o ritmo de sucção, o tempo da pausa entre os grupos de sucção e a coordenação entre sucção/deglutição/respiração.

Também foi observado se o bebê "mastigava" o mamilo, e se apresentava estalos de língua durante a sucção nutritiva.

Ao término da avaliação, as fotos e filmagens foram analisadas pelas três fonoaudiólogas.

Este estudo foi aprovado pelo Comitê de Ética em Pesquisa da Faculdade de Odontologia de Bauru da Universidade de São Paulo, sob o número $113 / 2011$.

\section{RESULTADO}

Dos 303 artigos identificados inicialmente pelo título e resumo, 85 foram selecionados para leitura na íntegra. Desses 26 foram incluídos nessa revisão. Também foram utilizados 2 livros e uma dissertação de mestrado.

O protocolo de avaliação do frênulo da língua em bebês foi elaborado considerando-se metodologias descritas em vários estudos ${ }^{5,7,8,11,13-19}$ e estudo piloto realizado em 10 bebês nascidos a termo, exposto na Figura 1. 
HISTÓRIA CLÍNICA

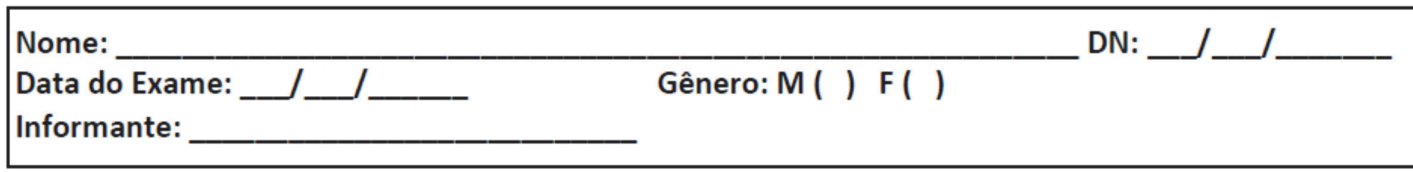

Endereço:

Bairro: Cidade/Estado: No:

Fones: Residencial: (__) Trabalho:(_) CEP:

Endereço eletrônico:

Nome do pai:

Nome da mãe:

Antecedentes Familiares (investigar se existem casos na família com alteração de frênulo da língua)

( ) não ( ) sim Quem e qual o problema:

Problemas de Saúde ( ) não ( )sim Quais:

Amamentação:

- tempo entre as mamadas: ( ) $3 \mathrm{~h}$ ( ) entre $1 \mathrm{~h}$ e $2 \mathrm{~h}(\mathrm{)}) \mathrm{1} / 2 \mathrm{~h}$ ou menos

- cansaço para mamar? ( ) não ( ) sim

- dor nos mamilos? ( ) não ( ) sim

- ferimento nos mamilos? ( ) não ( ) sim

A criança está com dificuldade de sugar?

() não () sim Se sim qual(is) dificuldade(s)?

\section{EXAME CLÍNICO}

\section{PARTE I - AVALIAÇÃO ANATOMOFUNCIONAL}

1. Postura de lábios em repouso

Celular:(

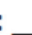
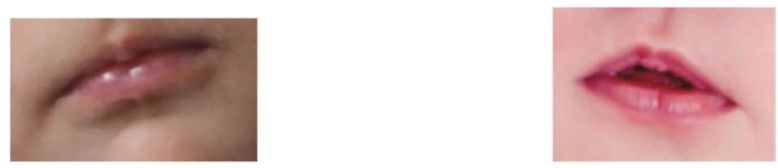

( ) lábios fechados $(0)$

( ) lábios entreabertos (1)

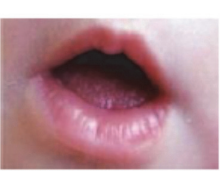

( ) lábios abertos (2)

2. Tendência do posicionamento da língua durante o choro
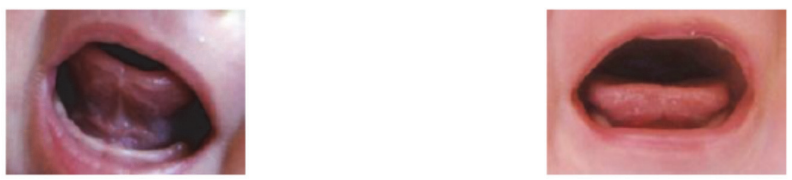

( ) língua elevada (0)

( ) língua na linha média (1)

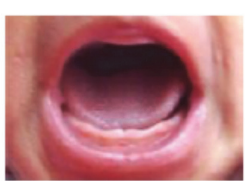

( ) língua baixa (2)

3. Frênulo da língua

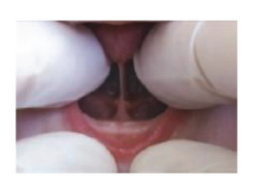

) é possível visualizar

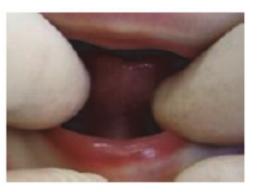

( ) não é possível visualizar

\section{NO CASO DE NÃO OBSERVÁVEL VÁ PARA O ITEM 4}


3.1. Espessura do frênulo

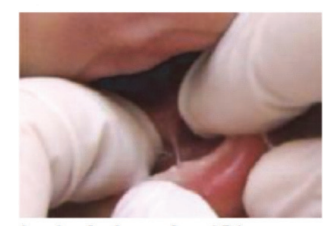

( ) delgado $(0)$

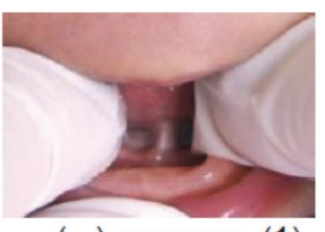

( ) espesso (1)

3.2. Fixação do frênulo na face sublingual (ventral) da língua

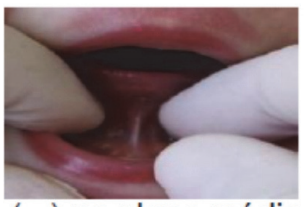

( ) no plano médio (0)

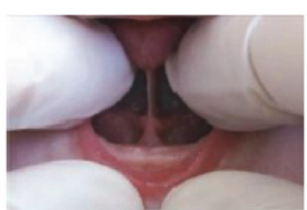

( ) entre o plano médio e o ápice (1)

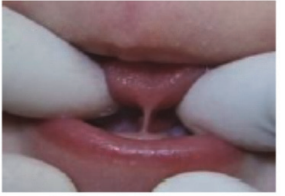

( ) no ápice (2)

3.3. Fixação do frênulo no assoalho da boca

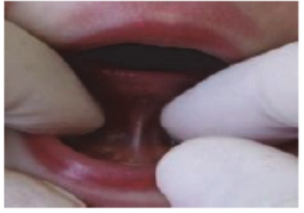

( ) visível a partir das carúnculas sublinguais (0) (abertura dos ductos submandibulares direito e esquerdo)

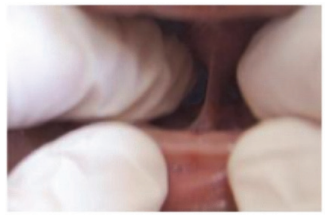

( ) visível a partir da crista alveloar inferior (1)

4. Forma da língua quando elevada durante o choro

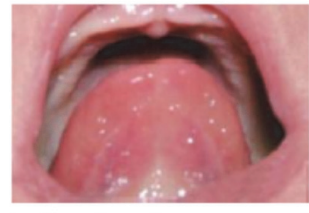

( )redonda ou quadrada (0)

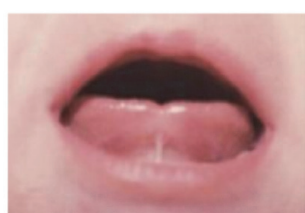

( ) ligeira fenda na ponta (1)

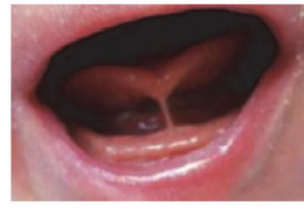

( ) formato de "coração" (2)

\section{PARTE II - AVALIAÇÃO DAS FUNÇÕES OROFACIAIS}

1. Sucção não nutritiva (sucção do dedo mínimo enluvado)

1.1. Coordenação do movimento da língua

( ) adequada: movimento coordenado (0) ( ) inadequada: movimento incoordenado (1)

(movimento ântero-posterior de língua)

\subsection{Canolamento da língua}

( ) presente $(0)$

(elevação das margens laterais e presença de sulco na região central da língua)

\subsection{Força da sucção}

\section{( ) forte (0)}

(forte compressão contra o palato, encontrando resistência

à retirada do dedo do avaliador da cavidade oral)
( ) fraca (1)

(fraca compressão contra o palato, encontrando pouca ou nenhuma resistência à retirada do dedo do avaliador da cavidade oral) 


\begin{tabular}{|lll|}
\hline 2. Sucção nutritiva (uso de oximetro) & Início: & Final: \\
Frequência cardíaca: & Início: & Final:_ bpm \\
Nivel de saturação de oxigênio: & bo
\end{tabular}

\subsection{Características do mamilo}

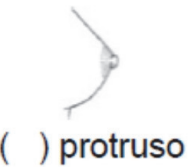

2.2. Pega do mamilo

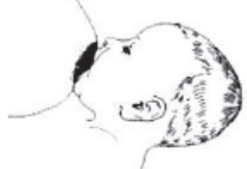

( ) abre bem a boca e abocanha o mamilo e parte da aréola (0)
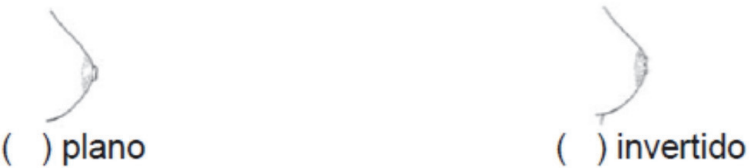

2.3. Sucção (observar o bebê mamando durante 5 minutos)

2.3.1. Ritmo da sucção (média de 3 grupos de sucção/pausa)

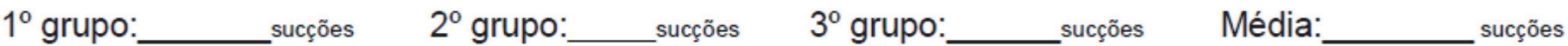

2.3.2. Tempo da pausa entre 3 grupos de sucção (uso de cronômetro)

$1^{\circ}$ grupo:____ segundos $2^{\circ}$ grupo:___ segundos $3^{\circ}$ grupo:___ segundos Média:___ segundos

\subsubsection{Coordenação entre sucção/deglutição/respiração}

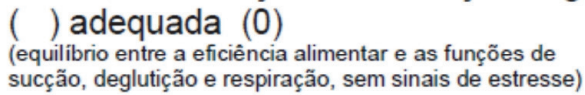

\subsection{4. "Mastiga" o mamilo}
( ) não (0)
( ) $\operatorname{sim}(1)$

2.3.5. Estalos de língua durante a sucção
() nenhum $(0)$
( ) assistemáticos (1)
( ) frequentes (2)

Figura 1 - Protocolo de avaliação de frênulo lingual em bebês

Quanto à aplicabilidade, esse instrumento se mostrou sensível para todos os itens propostos.

A primeira parte do protocolo é composta pela história clínica, onde constam os seguintes itens: data do exame, nome completo, gênero, data de nascimento, idade, endereço, telefone, nome dos pais, nome e grau de parentesco do informante, antecedentes familiares com alteração de frênulo lingual, dados sobre a saúde geral atual do bebê, verificação de intercorrências durante a amamentação como dor e/ou ferimentos nos mamilos ou alguma dificuldade, tempo entre as mamadas e presença de cansaço para mamar.
A segunda parte do protocolo é composta por uma avaliação anatomofuncional para observar aspectos gerais do frênulo da língua e uma avaliação das funções orofaciais para investigar os movimentos e a posição da língua na cavidade oral e as funções de sucção e deglutição durante a amamentação.

Para a avaliação anatomofuncional são propostos registros fotográficos e audiovisuais para análise posterior. Deve ser marcado o item que corresponde ao achado anatômico. É observada a postura dos lábios em repouso (que poderão estar vedados, entreabertos ou abertos); a tendência do 
posicionamento da língua durante o choro (elevada, na linha média ou abaixada). Por meio da elevação das margens laterais da língua com os dedos indicadores direito e esquerdo enluvados da avaliadora, é observada a possibilidade de visualizar ou não o frênulo ${ }^{8,13,16-18 ; ~ s e ~ f o r ~ p o s s i ́ v e l ~ v i s u a l i z a ́-l o, ~ e ́ ~}$ verificada sua espessura ${ }^{17}$ (delgado ou espesso). Também é observado se a fixação do frênulo na face sublingual (ventral) da língua se encontra no plano médio, entre o plano médio e o ápice ou no ápice ${ }^{8}$ e se a fixação no assoalho da boca é visível a partir das carúnculas sublinguais (abertura dos ductos submandibulares direito e esquerdo) ou a partir da crista alveolar inferior ${ }^{8,13}$.

$\mathrm{Na}$ avaliação das funções orofaciais são avaliadas a sucção não nutritiva e a sucção nutritiva. São realizados registros audiovisuais da sucção nutritiva para análise posterior.

A avaliação da sucção não nutritiva é avaliada com a introdução do dedo mínimo enluvado na boca do bebê para sugar durante 2 minutos ${ }^{24}$. É observada se a movimentação da língua ocorre de forma coordenada, com movimentos ântero-posteriores de língua ou incoordenada ${ }^{24}$; se o canolamento da língua está presente (ocorrendo a elevação das margens laterais e presença de sulco na região central da língua), ocorre pouco ou está ausente ${ }^{13,24}$. É verificada a força da sucção, e classificada como forte (quando houver compressão com força contra o palato, encontrando resistência à retirada do dedo do avaliador da cavidade oral) ou fraca (quando houver pouca ou nenhuma resistência à retirada do dedo do avaliador) ${ }^{24}$.

Durante a avaliação da sucção nutritiva, é sugerida a utilização de um oxímetro de pulso para medir a frequência cardíaca e o nível de saturação de oxigênio no sangue no início e no final da avaliação, para verificar se ocorrem alterações dos sinais vitais. É necessário que a avaliação da sucção nutritiva ocorra próxima à hora da mamada.

As características do mamilo materno são observadas, e o mesmo poderá ser classificado em protruso, plano ou invertido. Quanto à "pega" do mamilo é verificado se o bebê abre bem a boca e abocanha o mamilo e parte da aréola ou se pega apenas o mamilo.

$\mathrm{Na}$ avaliação da sucção propriamente dita, é observado o ritmo de sucção, contando-se o número de sucções ocorridas em três grupos de sucção separados por pausa e tirando-se a média. O tempo da pausa é cronometrado considerando o tempo das pausas entre três grupos de sucções, tirando-se em seguida, a média. A coordenação entre sucção/deglutição/respiração é considerada como adequada (quando houver equilíbrio entre a eficiência alimentar e as funções de sucção, deglutição e respiração, sem sinais de estresse) ou inadequada (se o bebê apresentar tosse, engasgos ou dispnéia $)^{24}$.

Também é observado se o bebê "mastiga" ou não o mamilo ${ }^{16}$, e se apresenta ou não estalos de língua durante a sucção nutritiva. Caso apresente os estalos, é verificado se os mesmos são assistemáticos ou frequentes.

O protocolo contém escores, com escala progressiva de pontuação, onde zero significa a normalidade, enquanto a pontuação um e dois, em ordem crescente, indicam características de alteração.

\section{DISCUSSÃO}

Na literatura pesquisada, foi encontrado apenas um protocolo específico, mas não validado para avaliação do frênulo da língua em bebês, considerando aspectos anatômicos e de mobilidade da

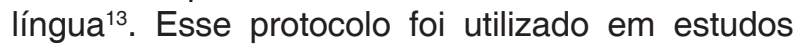
posteriores, havendo controvérsias na sua exequibilidade e efetividade ${ }^{11,14-15}$. Outros estudos propõem a inspeção visual do frênulo da língua e o levantamento dos sinais e sintomas apresentados pelo bebê e sua mãe durante a amamentação. Dor e ferimento nos mamilos e dificuldade na pega foram os sinais e sintomas mais relacionados à alteração do frênulo $7,16-19$. Um estudo utilizou um protocolo específico para avaliação do frênulo da língua em indivíduos maiores de 7 anos, considerando tanto aspectos anatômicos do frênulo e da língua, quanto os movimentos da língua e a fala ${ }^{8}$.

Embora haja falta de consenso quanto à classificação das alterações de frênulo, atualmente muitos estudos confirmam a interferência dessas alterações na amamentação de bebês, ${ }^{511,13-23}$.

Por avaliar bebês, a testagem deve ser breve, não invasiva, apresentar baixo risco para o bebê e identificar os sinais e sintomas de uma possível interferência das alterações do frênulo na amamentação.

A elaboração do protocolo aqui apresentado leva em consideração o fato de não existirem propostas que avaliem simultaneamente, as características da língua e do frênulo e as funções de sucção e deglutição durante a amamentação. Este protocolo, inicialmente, visa levantar dados sobre normalidade e alteração das funções, correlacionando-as com o frênulo, para possíveis intervenções precoces, minimizando ou eliminando futuras alterações nas funções orofaciais de mastigação e fala.

Foram encontradas na literatura, divergências de opiniões quanto às consequências de um frênulo alterado, bem como a necessidade de intervenção cirúrgica. As frenectomias ou frenotomias 
do frênulo, até hoje, são motivo de muita discussão sobre a necessidade ou não da intervenção, quando deve ser realizada, qual a melhor técnica e qual profissional seria habilitado para realizar o procedimento $5,16,19,22,25-29$.

Espera-se que a somatória da avaliação de uma série de aspectos da língua e do frênulo, considerando forma, fixação, espessura e da avaliação das possibilidades de movimentos e das funções possam contribuir para um diagnóstico preciso. A existência de um protocolo consistente, aplicado por diferentes avaliadores, poderá diminuir o número de controvérsias sobre as possíveis alterações do frênulo da língua e sua interferência nas funções orofaciais, auxiliando na indicação ou não de procedimento cirúrgico.

Basear-se em protocolos específicos permite atuar de forma planejada, documentando procedimentos, consolidando e sustentando com evidências as práticas clínicas.

\section{CONCLUSÃO}

O protocolo de avaliação do frênulo da língua em bebês com escores é baseado numa proposta teórica e num projeto piloto com 10 sujeitos; depende, portanto, de sua aplicabilidade em um número maior de bebês e análise estatística para se configurar como um teste validado. A proposta apresentada pode auxiliar os profissionais da saúde a avaliar e diagnosticar as variações anatômicas do frênulo e sua possível interferência na amamentação norteando condutas eficazes, e promovendo uma prática baseada em evidências. A segunda fase deste trabalho compreenderá a pesquisa experimental e a análise estatística dos dados.

\begin{abstract}
Purpose: to present a protocol proposal to evaluate the lingual frenum in babies with scores. Method: initially was performed a review of the literature, referring to the databases Web of Science, Pubmed, Embase, All Evidence-Based Medicine Reviews and Scielo as well as textbooks in the areas of Anatomy, Pediatric Dentistry and Language and Hearing Sciences. All aspects considered relevant were included in the work found in the initial release, which was assessed on three Speech Language Pathologists specialist in Orofacial Myology, obtaining the consensus version, which was applied in 10 term infants to verify the applicability of the instrument. Results: based on literature and the pilot study, the protocol was designed to evaluate the lingual frenum in infants. The first part consists of the clinical history containing general questions of identification and specific questions about family history and breastfeeding. The second part is composed by clinical examination, that consists of anatomofunctional evaluation and evaluation of orofacial functions. Conclusions: the evaluation protocol of the frenum of the tongue in infants has been developed from a theoretical proposal and depends on its applicability to be configured as validated test. It is hoped that it may help health professionals to assess and diagnose the anatomical variations of the frenum and its possible interference with breast feeding, guiding and promoting effective conduct an evidence-based practice. The second phase of this work will be the experimental research and statistical analysis.
\end{abstract}

KEYWORDS: Evaluation; Protocols; Lingual Frenum; Breastfeeding; Suction; Speech, Language and Hearing Sciences

\section{REFERÊNCIAS}

1. Mosby's medical, nursing, e allied health dictionary/revision editor, Kenneth NA. $5^{\text {a }}$ ed. St Louis, Missouri; Mosby, Inc; 1998.

2. Singh S, Kent RD. Dictionary of speech-language pathology. San Diego, California: Singular's; 2000.

3. Lalakea ML, Messner AH. Ankyloglossia: the adolescent and adult perspective. Otolaryngol Head Neck Surg 2002;127:539-45.
4. Elias Podesta MC, Nunez Del Arco MS, Tello Meléndez PG, Cháves González BA. Diagnóstico clínico de anquiloglossia, posibles complicaciones y propuesta de solución quirurgica. Gac Odontol 2001;3:13-7.

5. Messner AH, Lalakea ML, Aby J, MacMahon J, Bair E. Ankyloglossia incidence and associated feeding difficulties. Arch Otolaryngol Head Neck Surg 2000;126:36-9. 
6. Messner $\mathrm{AH}$, Lalakea ML. The effect of ankyloglossia on speech in children. Otolaryngol Head Neck Surg 2002;127:539-45.

7. Kotlow LA. Ankiloglossia (tongue-tie): a diagnostic and treatment quandary. Quintessence Int 1999;31:276-8.

8. Marchesan IQ. Protocolo de avaliação do frênulo da língua. Rev CEFAC 2010;12(6):977-89.

9. Voros-Balog T, Vincze N, Banoczy J. Prevalence of tongue lesions in Hungarian children. Oral Dis: 2003;9:84-7.

10. Sedano HO, Carreon Freyre I, Garza de la Garza ML, Gomar Franco CM, Grimaldo Hernandez C, Hernandez Montoya ME, Hipp C, Keenan KM, Martinez Bravo J, Medina López JA. Clinical orodental abnormalities in Mexican children. Oral Surg Oral Med Oral Pathol: 1989;68:300-11.

11. Ballard JL, Auer CE, Khoury JC. Ankyloglossia: assessment, incidence, and effect of frenuloplasty on the breastfeeding dyad. Pediatrics 2002;110(5):1-6. 12. Marchesan IQ. Lingual frenulum: quantitative evaluation proposal. Int $\mathrm{J}$ Orofacial Myology; 2005;31:39-48.

13. Hazelbaker AK. The assessment tool for lingual frenulum function (ATLFF): Use in a lactation consultant private practice. Pasadena, CA: Pacific Oaks College; 1993. Thesis.

14. Ricke LA, Baker NJ, Madlon-Kay DJ, et al. Newborn tongue tie: prevalence and effect on breast-feeding. J Am Board Fam Pract 2005;18:1-7. 15. Kupietzky A, Botzer E. Ankyloglossia in the Infant and Young Child: Clinical Suggestions for Diagnosis and Management. Pediatric Dentistry. 2005;27:1.

16. Knox I. Tongue Tie and Frenotomy in the Breastfeeding Newborn. NeoReviews 2010; 11(9):513-9.

17. Coryllos E, Watson Genna C, Salloum AC. Congenital tongue-tie and its impact in breastfeeding. Breastfeeding: Best for mother and baby Newsletter 2004;1-6.

18. Griffiths DM. Do Tongue Ties Affect Breastfeeding? J Hum Lact 2004;20(4):409-14.
19. Hogan M, Westcott C, Griffiths M. Randomized, controlled trial of division of tongue tie in infants with feeding problems. J Paediatr Child Health 2005;41:246-50.

20. Hall DMB, Renfrew MJ. Tongue-tie: common problem or old wives tale. Arch Dis Child 2005; 90:1211-5.

21. Segal LM, Stephenson R, Dawes M, Feldman P. Prevalence, diagnosis, and treatment of ankyloglossia. Can Fam Physician 2007;53:1027-33. 22. Geddes DT, Langton DB, Gollow I, Jacobs LA, Hartmann PE, Simmer K. Frenulotomy for Breastfeeding Infants With Ankyloglossia: Effect on Milk Removal and Sucking Mechanism as Imaged by Ultrasound. Pediatrics. 2008;122:e188-e94.

23. Geddes DT, Kent JC, McClellan HL, Garbin CP, Chadwick LM, Hartmann PE. Sucking characteristics of successfully breastfeeding infants with ankyloglossia: a case series. Acta Pædiatrica. 2010;99:301-3.

24. Fujinaga $\mathrm{Cl}$, Scochi CGS, Santos CB, Zamberlan NE, Leite AM. Validação do conteúdo de um instrumento para avaliação da prontidão do prematuro para início da alimentação oral. Rev Bras Saúde Matern Infant. 2008;8(4):391-9.

25. Suter VG, Bornstein MM. Ankyloglossia: facts and myths in diagnosis and treatment. J Periodontol. 2009 Aug;80(8):1204-19.

26. Miranda BH, Milroy CJ. A quick snip - A study of the impact of outpatient tongue tie release on neonatal growth and breastfeeding. J Plast Reconstr Aesthet Surg 2010;63(9):e683-5.

27. Navarro NP, López M. Anquiloglossia en niños de 5 a 11 años de edad. Diagnóstico y tratamiento. Rev Cubana Estomatol. 2002;39(3):3-7.

28. Wallace $\mathrm{H}$, Clarke $\mathrm{S}$. Tongue tie division in infants with breast-feeding difficulties. Int $\mathrm{J}$ Pediatr Oto. 2006; 70(7):1257-61.

29. Tuli A, Singh A. Monopolar diathermy used for correction of ankyloglossia.J Indian Soc Pedod Prev Dent 2010;28:130-3.

RECEBIDO EM: 03/10/2011

ACEITO EM: 15/01/2012

Endereço para correspondência:

Roberta Lopes de Castro Martinelli

Avenida Ângelo Piva, 358 - Centro

Brotas - São Paulo

CEP:17380-000

E-mail: robertalcm@gmail.com 